\title{
Segmental resection is associated with decreased survival in patients with stage IA non-small cell lung cancer with a tumor size of 21-30 $\mathrm{mm}$
}

\author{
Xiangyang Yu ${ }^{1 \#}$, Rusi Zhang ${ }^{1 \#}$, Mengqi Zhang ${ }^{2 \#}$, Yongbin Lin ${ }^{1}$, Xuewen Zhang ${ }^{3}$, Yingsheng Wen ${ }^{1}$, \\ Longjun Yang ${ }^{1}$, Zirui Huang ${ }^{1}$, Gongming Wang ${ }^{1}$, Dechang Zhao ${ }^{1}$, Michel Gonzalez ${ }^{4}$, Jean-Marc Baste ${ }^{5}$, \\ Rene Horsleben Petersen ${ }^{6}$, Calvin S. H. Ng ${ }^{7}$, Alessandro Brunelli ${ }^{8}$, Lie Zheng ${ }^{9}$, Lanjun Zhang ${ }^{1}$; \\ written on behalf of the AME Thoracic Surgery Collaborative Group
}

${ }^{1}$ State Key Laboratory of Oncology in South China, Collaborative Innovation Center for Cancer Medicine, Department of Thoracic Surgery, Sun Yat-sen University Cancer Center, Guangzhou, China; ${ }^{2}$ Department of Pathology, Shenzhen Maternity and Child Healthcare Hospital, Shenzhen, China; ${ }^{3}$ State Key Laboratory of Oncology in South China, Collaborative Innovation Center for Cancer Medicine, Department of Anesthesiology, Sun Yat-sen University Cancer Center, Guangzhou, China; ${ }^{4}$ Service of Thoracic Surgery, University Hospital of Lausanne, University of Lausanne, Lausanne, Switzerland; ${ }^{5}$ Department of General and Thoracic Surgery, Rouen University Hospital, Rouen, France; ${ }^{6}$ Department of Cardiothoracic Surgery, University Hospital of Copenhagen, Rigshospitalet, Copenhagen, Denmark; ${ }^{7}$ Department of Surgery, The Chinese University of Hong Kong, Prince of Wales Hospital, Shatin, New Territories, Hong Kong, China; ${ }^{8}$ Department of Thoracic Surgery, St James's University Hospital, Leeds, UK; 'State Key Laboratory of Oncology in South China, Collaborative Innovation Center for Cancer Medicine, Department of Medical Imaging, Sun Yat-sen University Cancer Center, Guangzhou, China

Contributions: (I) Conception and design: X Yu, L Zheng, L Zhang, CSH Ng; (II) Administrative support: R Zhang, M Zhang, Y Lin, X Zhang, Y Wen, L Yang, M Gonzalez; (III) Provision of study materials or patients: X Yu, Z Huang, G Wang, D Zhao; (IV) Collection and assembly of data: X Yu, R Zhang, M Zhang, Y Lin, X Zhang, L Zheng, L Zhang; (V) Data analysis and interpretation: X Yu, R Zhang, M Zhang, M Gonzalez, JM Baste, RH Petersen, CSH Ng, A Brunelli; (VI) Manuscript writing: All authors; (VII) Final approval of manuscript: All authors.

\#These authors contributed equally to this work.

Correspondence to: Lanjun Zhang, MD, PhD. Department of Thoracic Surgery, Sun Yat-sen University Cancer Center, 510021 Guangzhou, China. Email: zhanglj@sysucc.org.cn; Lie Zheng, MD. Department of Medical Imaging, Sun Yat-sen University Cancer Center, 510021 Guangzhou, China. Email: zhengl@sysucc.org.cn.

Background: The feasibility of segmental resection for early-stage non-small cell lung cancer (NSCLC) is still controversial. This study aimed to compare survival outcomes following lobectomy and segmental resection in patients with pathological T1cN0M0 (tumor size 21-30 mm) NSCLC.

Methods: Patients diagnosed between 1998 and 2016 with pathological stage IA NSCLC and with tumors measuring 21-30 mm were extracted from the Surveillance, Epidemiology, and End Results (SEER) database. The observational outcomes were cancer-specific survival (CSS) and overall survival (OS) at 5 years. Univariate survival analysis was carried out to identify potential prognostic factors of prolonged survival. Cox proportional hazards model was used to adjust for confounding factors. Additionally, pairwise comparisons were conducted between lobectomy and segmental resection for CSS and OS, and forest plots were drawn.

Results: Of the 9,580 patients analyzed, 400 patients (4.2\%) underwent segmental resections. Patients with older age $(\mathrm{P}<0.001)$, smaller tumors $(\mathrm{P}<0.001)$, and left-sided tumors $(\mathrm{P}=0.002)$ were more likely to receive segmental resection. No difference was found in the operative mortality rates between the segmental resection group and the lobectomy group (1.0\% vs. 1.2\%, $\mathrm{P}=0.707)$. The CSS (HR, 1.429; 95\% CI, $1.166-$ 1.752; $\mathrm{P}=0.001)$ and $\mathrm{OS}(\mathrm{HR}, 1.348 ; 95 \% \mathrm{CI}, 1.176-1.544 ; \mathrm{P}<0.001)$ in the segmental resection group were significantly worse than those in the lobectomy group. Subgroup analyses by age, year of diagnosis, sex, tumor size, histology, grade, and the number of dissected lymph nodes also confirmed that lobectomy was associated with improved CSS and OS.

Conclusions: Lobectomy and thorough removal of lymph nodes should continue to be the recommended standard of care for patients with surgically resectable stage IA NSCLC with tumor size of 21-30 mm. 
Keywords: Early-stage; segmental resection; lobectomy; Surveillance, Epidemiology, and End Results (SEER)

Submitted Jul 20, 2020. Accepted for publication Jan 14, 2021.

doi: $10.21037 /$ tlcr-20-1217

View this article at: http://dx.doi.org/10.21037/tlcr-20-1217

\section{Introduction}

In 2016, the International Association for the Study of Lung Cancer (IASLC) Staging Committee published the eighth edition of the Tumor, Node, and Metastasis (TNM) classification for lung cancer. Since its introduction in January 2017 , it has been widely used in clinical practice (1). To allow accurate diagnosis of early-stage lung cancer, stage IA $(\leq 30 \mathrm{~mm})$ is divided into three subsets: stage IA1 $(\leq 10 \mathrm{~mm}$, T1aN0M0), stage IA2 (>10-20 mm, T1bN0M0), and stage IA3 (>20-30 mm, T1cN0M0) (1). These new cutoff points may also serve a crucial role in determining the extent of surgical resection (2-4).

A randomized controlled trial (RCT) conducted by the Lung Cancer Study Group in 1995 compared lobectomy with intentional sublobar resection (i.e., anatomical segmental resection and non-anatomical wedge resection) for patients with peripheral T1N0 $(\leq 30 \mathrm{~mm})$ non-small cell lung cancer (NSCLC). This study reported that, compared with lobectomy, intentional sublobar resection did not improve postoperative morbidity, mortality, or pulmonary function, but increased the risks of locoregional recurrence and cancer-specific death (5). Therefore, lobectomy has been the standard surgical procedure for stage I $(\leq 30 \mathrm{~mm})$ NSCLC for the past 25 years (6). However, numerous retrospective investigations have reported the survival outcomes of segmental resection and lobectomy to be similar in patients with peripheral small-sized NSCLC $(\leq 20 \mathrm{~mm})$; moreover, patients who underwent segmental resection appeared a higher perioperative quality of life (QoL) than those who underwent lobectomy for earlystage NSCLC $(2,4)$. In addition, two ongoing multicenter phase III RCTs [Cancer and Leukemia Group B (GBCALGB-140503) and the Japan Clinical Oncology Group/West Japan Oncology Group (JCOG0802/ WJOG4607L)] comparing segmental resection with lobectomy for clinical T1N0M0 $(\leq 20 \mathrm{~mm})$ NSCLC are expected to provide high-level evidence for thoracic surgeons to assess whether intentional segmental resection could be the new standard of care for stage T1a-b NSCLC without lymph node involvement or distant metastasis (7).
Moreover, the development of new technologies, such as fluorescence, three-dimensional reconstruction/printed models, may help clinicians overcome technical obstacles (anatomical variations, intersegmental plan, margins etc.), allowing them to perform safe segmentectomies and simplify the surgical procedure (8).

Notably, stage IA NSCLC with a tumor size of $>20-30 \mathrm{~mm}$ has not been included in the inclusion criteria of the RCTs mentioned above. In addition, few retrospective studies have assessed the potential utility of segmental resection for this specific population $(3,9)$. In a comparison of propensityscore matched cohorts (37 pairs), Kamigaichi et al. observed no significant differences in recurrence-free survival (RFS) or overall survival (OS) for solid-dominant clinical stage IA lung tumors measuring 21-30 mm when lobectomy and segmental resection were compared (3). On the contrary, in a Surveillance, Epidemiology, and End Results (SEER) data analysis of patients with pathological stage I NSCLC, the subgroup analysis for tumors measuring $21-30 \mathrm{~mm}$ as defined under the 7th edition staging system (T1b) demonstrated that lobectomy was associated with significantly better OS (10). Therefore, the optimal the extent of surgical resection for stage T1cN0M0 (>20-30 mm) NSCLC, as defined by the 8 th edition staging proposal, is still unclear.

In this study, we used data from the SEER database to evaluate whether lobectomy confers a survival advantage over anatomic segmental resection in patients with pathological T1cN0M0 (21-30 mm) NSCLC. And we present the following article in accordance with the Strengthening the Reporting of Observational Studies in Epidemiology (STROBE) reporting checklist (available at http://dx.doi.org/10.21037/tlcr-20-1217).

\section{Methods}

\section{Patient selection}

Data of patients who underwent segmental resection (surgery of primary site code: 22) and lobectomy (surgery of primary site codes: 30 and 33) for primary lung cancer between 1998 and 2016 were obtained from the SEER 18 Regs Custom 
Surveillance, Epidemiology, and End Results (SEER) 18 Regs Custom Data (with additional treatment fields), November 2018 Submission (1975-2016 varying)

International Classification of Disease for Oncology, 3rd edition, Site Recode: lung and bronchus;

Site and Morphology Behavior Recode for Analysis: malignant;

Year of Diagnosis: 1998-2016;

Therapy.RX Summ-Surg Prim Site: 22, 30, 33.

$(\mathrm{N}=134,184)$

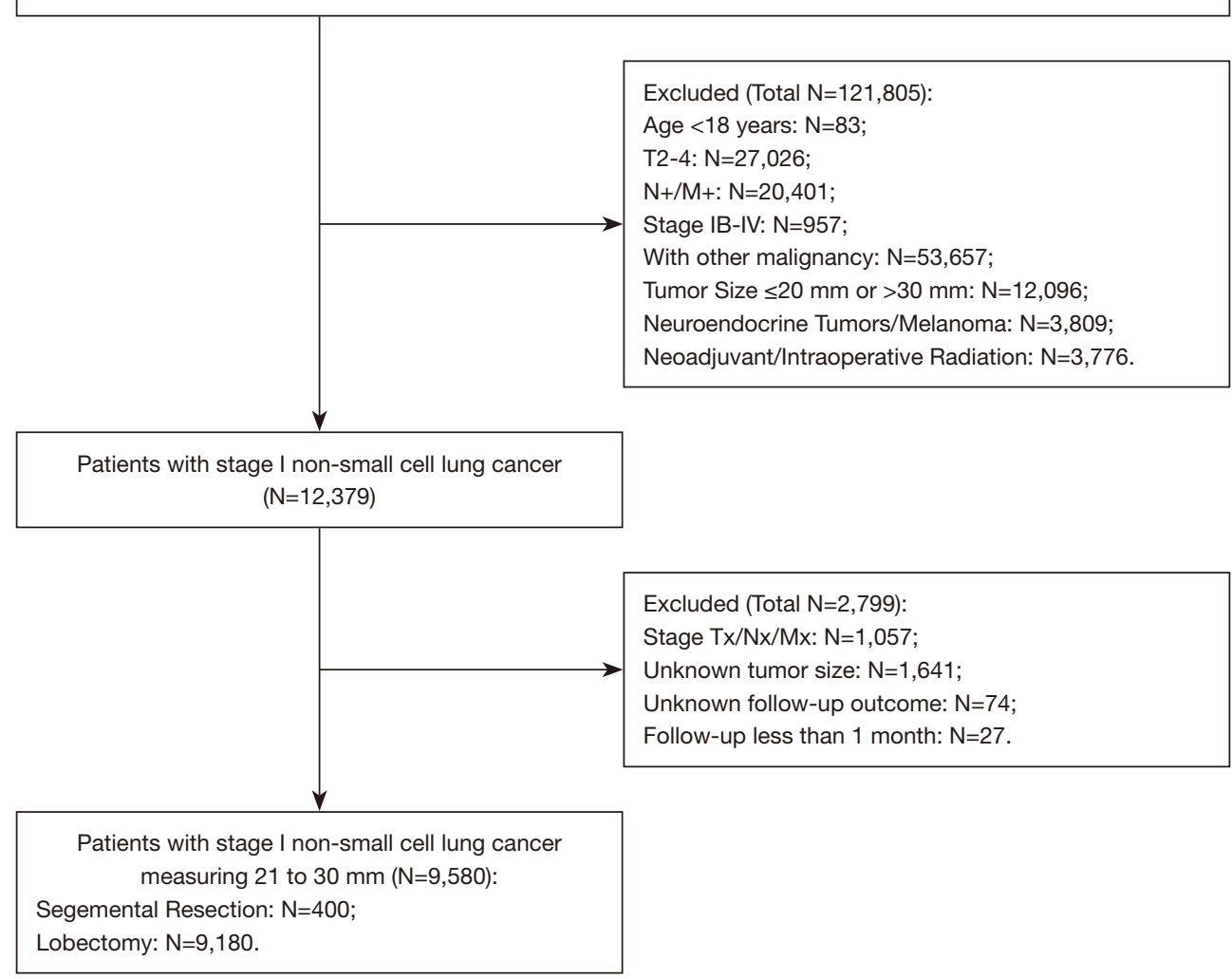

Figure 1 Screening process of the study population. All patients were restaged according to the eighth edition of the TNM staging system.

Data (with additional treatment fields). Patients aged $<18$ years, with additional synchronous malignancies, diagnosed with pulmonary neuroendocrine tumors or melanoma (i.e., carcinoid, large cell neuroendocrine tumor, small cell lung cancer), who received neoadjuvant or intraoperative therapy, or who had incomplete clinicopathological or follow-up information, were excluded. In addition, since this study focused on patients with stage IA NSCLC with a tumor size of $>20-30 \mathrm{~mm}$,patients with nodal involvement, distant metastases, or a tumor size $\leq 20 \mathrm{~mm}$ or $>30 \mathrm{~mm}$ were also excluded from the final analysis. Figure 1 shows the detailed screening process.

\section{Ethical statement}

The study was conducted in accordance with the
Declaration of Helsinki (as revised in 2013). This retrospective study based on the SEER database was approved by the Surveillance Research Program in National Cancer Institute's Division of Cancer Control and Population Sciences (DCCPS) (No. 12101-Nov2018), and was also approved by the institutional review at the Sun Yatsen University Cancer Center (No. B2018-011). Because of the retrospective nature of the research, the requirement for informed consent was waived.

\section{Statistical analysis}

We used the X-tile software (Copyright Yale University 2003) to determine the optimal cutoff points. The continuous variables, including age, tumor size, and the number of dissected lymph nodes, were all transformed into 
categorical variables. The chi-square or Fisher's exact test were used to compare frequencies and percentages between different subgroups.

The observational outcomes of the present study were cancer-specific survival (CSS) and OS, which were measured in months. Patients who were still alive on November 31, 2019, were recorded as right-censored cases in the OS analyses. In the CSS analyses, right-censored cases also included patients who died of causes not related to lung cancer. Operative mortality was defined as any death within 30 days after surgery. In univariate survival analyses, the Kaplan-Meier method was applied, and the survival curves were plotted with the GraphPad Prism software (version 7, http://www.graphpad.com/) and compared using the log-rank test. Subsequently, the potential prognostic factors $(\mathrm{P}<0.05)$ for CSS and OS that were identified in the univariate analyses were included in the Cox proportional hazards models. Forest plots were drawn using the "forestplot" package in $\mathrm{R}$ software (version 3.5.3, https://www.r-project.org/).

All statistical analyses were conducted with SPSS software (version 24, IBM, NY, USA; https://www.ibm. com/), with a two-sided $\mathrm{P}$ value $<0.05$ set as the threshold for statistical significance.

\section{Results}

\section{Patient characteristics}

A total of 9,580 patients with stage IA NSCLC with a tumor size of 21-30 mm who met the inclusion criteria were identified from the SEER database. The clinicopathological variables of the lobectomy group $(9,180,95.8 \%)$ and the segmental resection group (400, 4.2\%) were compared (Table 1). Segmental resections were more likely to be performed in patients with older age ( $\geq 60$ : $86.5 \%$ ), smaller tumor size (21-25 mm: 64.8\%), or left-sided tumors (48.8\%); moreover, segmental resections became more popular in the later part of the study period (2008-2016: 52.5\%). More lymph nodes were removed in the lobectomy group than in the segmental resection group (median: $8.8 v s .4 .4, \mathrm{P}<0.001$ ). A higher proportion of patients in the segmental resection group received radiation after surgery $(3.5 \%$ vs. $1.6 \%, \mathrm{P}=0.004)$. The operative mortality rates between the two groups were not significantly different $(1.2 \%$ vs. $1.0 \%, \mathrm{P}=0.707)$.

\section{Survival analysis}

During follow-up (median: 48.0 months; range,
0-227 months), 2,075 patients died from lung cancer-specific causes, and 2,380 patients died due to other causes. The 5-year CSS and OS rates for stage IA $(21-30 \mathrm{~mm})$ patients were $84.1 \%$ and $63.6 \%$, respectively. In univariate survival analysis (Table 2), lobectomy was associated with significant survival benefit in both CSS and OS (median CSS, 165 vs. 119 months, $\mathrm{P}<0.001$; median OS, 94 vs. 61 months, $\mathrm{P}<0.001)$. Univariate analysis also revealed that age (Figure $2 A, B$ ), year of diagnosis, sex, race, tumor size (Figure 2C,D), histology, grade, number of dissected lymph nodes (Figure 2E,F), insurance, and marital status were significant prognostic factors for CSS and OS. Meanwhile, postoperative radiation and chemotherapy were associated with worse OS (all $\mathrm{P}<0.001$ ), but were not associated with worse CSS.

In the Cox proportional hazards analysis, lobectomy was consistently associated with significantly improved survival (Figure 2G,H; Table 3). Additionally, age $<60$ years, year of diagnosis after 2002, female sex, tumor size of 21-25 mm (but not for CSS), adenocarcinoma, well/moderately differentiated cancer (but not for CSS), >3 lymph nodes dissected, no postoperative radiation or chemotherapy (but not for CSS), any insurance (but not for CSS), and marriage were still significantly associated with longer survival.

\section{Subgroup analysis}

Forest plot analyses for age, year of diagnosis, sex, race, tumor size, histology, lymph node dissection, radiation, chemotherapy, insurance, and marital status showed that lobectomy for stage IA $(21-30 \mathrm{~mm})$ NSCLC was associated with improved CSS and OS in most subgroups (Figure 3). Notably, in the subgroup of patients aged $<60$ years or of the black race, the segmental resection group demonstrated comparable CSS [hazard ratio (HR), 0.974, 95\% confidence interval (CI): 0.401-2.366, $\mathrm{P}=0.954$; and $\mathrm{HR}, 1.014,95 \%$ CI: 0.414-2.480, $\mathrm{P}=0.976$; respectively] and OS (HR, 1.141, 95\% CI: 0.714-1.824, P=0.579; and HR, 1.053 , 95\% CI: $0.616-1.798, \mathrm{P}=0.850$, respectively) to the lobectomy group.

\section{Discussion}

This population-based study indicated that segmental resection was associated with decreased survival for patients with pathological stage IA NSCLC with a tumor size of 21-30 $\mathrm{mm}$. The potential survival benefits offered by lobectomy over segmental resection were confirmed in subgroup analyses of sex, histology, grade, tumor size, and the number of dissected lymph nodes. To our knowledge, 
Table 1 Baseline characteristics of patients with pathological stage IA (21-30 mm) non-small cell lung cancer following lobectomy and segmental resection

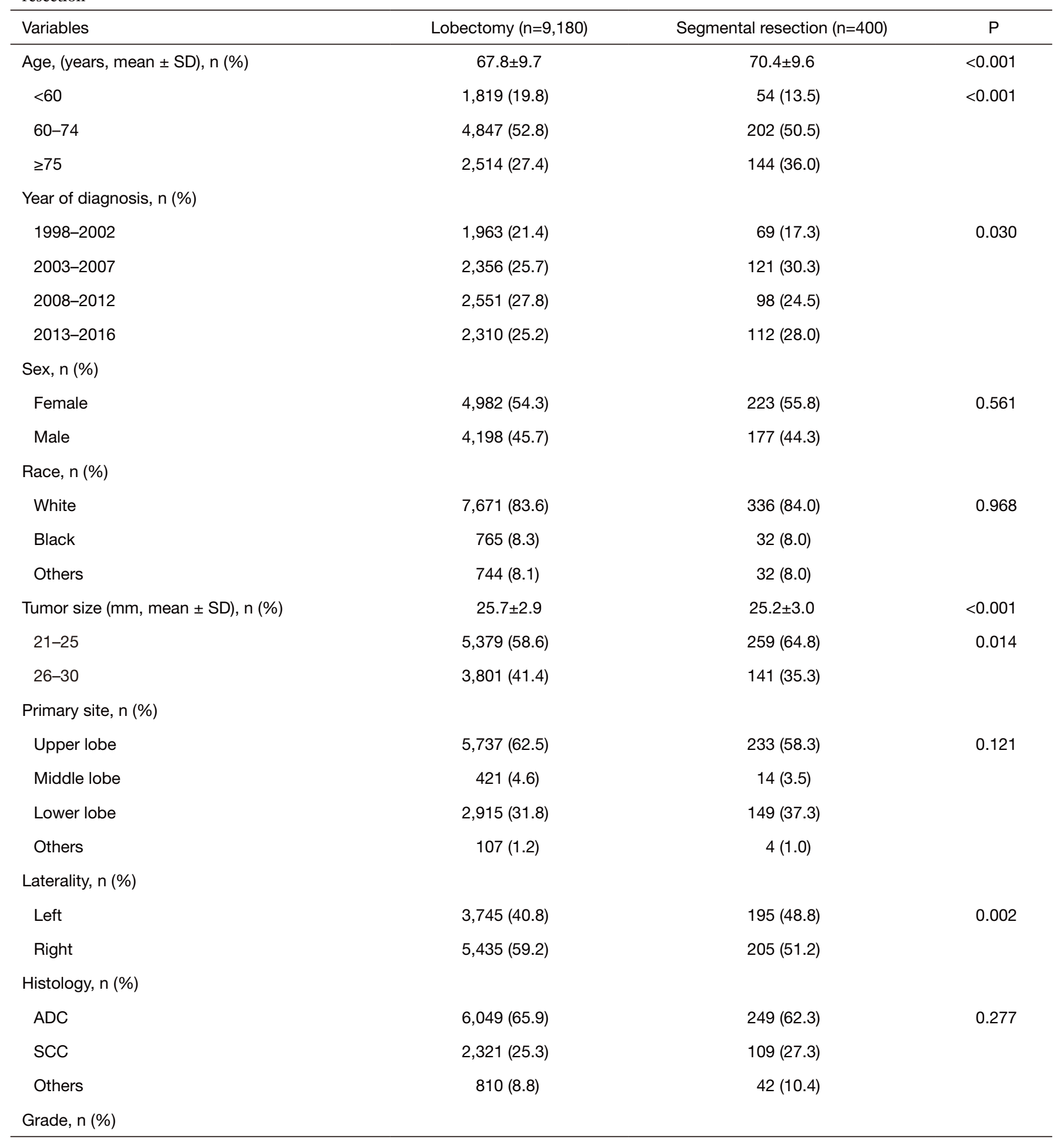

Table 1 (continued) 
Table 1 (continued)

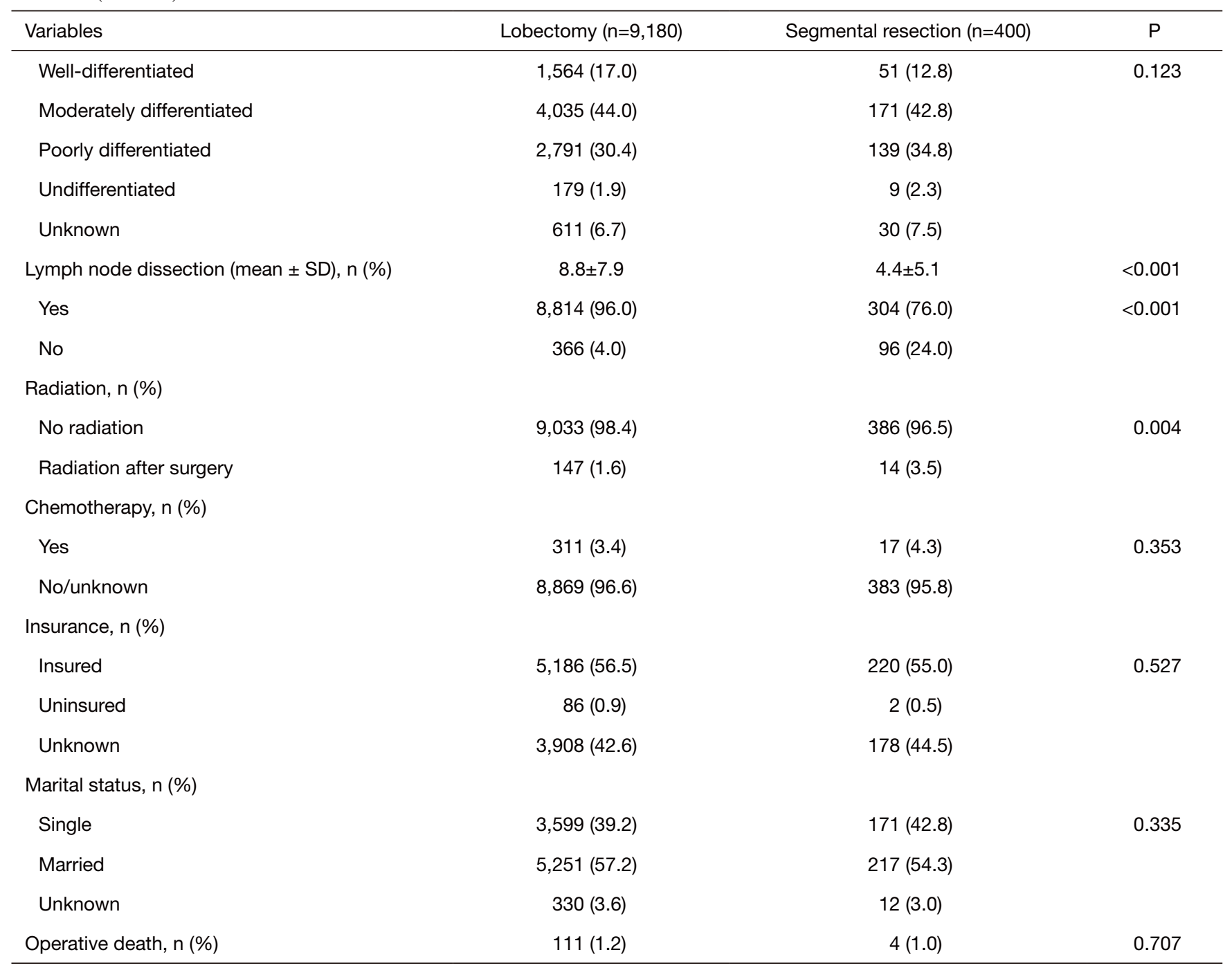

$\mathrm{SD}$, standard deviation; ADC, adenocarcinoma; SCC, squamous cell carcinoma.

this is the first study to compare the oncological outcomes of lobectomy and segmental resection for NSCLC patients staged as pT1cN0M0 according to the 8 th edition of the TNM Classification for Lung Cancer.

The extent of lung resection for patients with resectable stage IA NSCLC is still a debated issue. Compared with non-anatomical wedge resection, anatomical segmental resection is more likely to achieve safe surgical margins and to facilitate the evaluation of subsegmental and hilar lymph nodes $(11,12)$. Therefore, a number of studies have focused on comparing oncological outcomes between lobectomy and segmental resection but have excluded wedge resections for stage IA NSCLC $(2,3,9)$. A number of recent studies, which include retrospective propensity-score matched analyses and the SEER database analyses, have demonstrated that patients who undergo anatomical segmental resection may achieve outcomes comparable to those of patients who undergo lobectomy for stage IA NSCLC of $\leq 20 \mathrm{~mm}$ (2). However, there has been little research that has assessed the potential of segmental resection for stage IA NSCLC with tumors measuring $>20-30 \mathrm{~mm}$, perhaps because patients with cT1c (>20-30 mm) NSCLC have been reported to have a worse prognosis and higher risk of lymph node metastasis than those with cT1a-1b $(\leq 20 \mathrm{~mm})$ NSCLC $(3,9,13,14)$. Therefore, we hypothesized that a larger extent of lung resection (lobectomy) may be required to obtain the 
Table 2 Univariate survival analyses for cancer-specific survival and overall survival in patients with pathological stage IA (21-30 mm) non-small cell lung cancer

\begin{tabular}{|c|c|c|c|c|c|}
\hline Variables & No. & \multicolumn{2}{|c|}{ Cancer-specific survival (CSS) } & \multicolumn{2}{|c|}{ Overall survival (OS) } \\
\hline \multicolumn{6}{|l|}{ Age, years } \\
\hline$<60$ & 1,873 & 93.1 & $<0.001$ & 74.0 & $<0.001$ \\
\hline $60-74$ & 5,049 & 85.1 & & 64.8 & \\
\hline \multicolumn{6}{|c|}{ Year of diagnosis } \\
\hline 1998-2002 & 2,032 & 81.2 & $<0.001$ & 56.1 & $<0.001$ \\
\hline 2003-2007 & 2,477 & 84.3 & & 60.8 & \\
\hline 2008-2012 & 2,649 & 84.7 & & 68.3 & \\
\hline Female & 5,205 & 87.5 & $<0.001$ & 68.9 & $<0.001$ \\
\hline Male & 4,375 & 79.9 & & 57.2 & \\
\hline \multicolumn{6}{|l|}{ Race } \\
\hline White & 8,007 & 83.3 & $<0.001$ & 62.8 & $<0.001$ \\
\hline Black & 797 & 85.4 & & 61.8 & \\
\hline Others & 776 & 91.4 & & 73.3 & \\
\hline \multicolumn{6}{|c|}{ Tumor size, mm } \\
\hline $21-25$ & 5,638 & 85.2 & 0.001 & 65.8 & $<0.001$ \\
\hline Lower lobe & 3,064 & 83.6 & & 58.6 & \\
\hline Others & 111 & 79.5 & & 56.2 & \\
\hline \multicolumn{6}{|l|}{ Laterality } \\
\hline Left & 3,940 & 83.5 & 0.511 & 62.9 & 0.342 \\
\hline Right & 5,640 & 84.5 & & 64.0 & \\
\hline \multicolumn{6}{|l|}{ Histology } \\
\hline ADC & 6,298 & 87.5 & $<0.001$ & 68.2 & $<0.001$ \\
\hline SCC & 2,430 & 76.8 & & 55.5 & \\
\hline Others & 852 & 80.2 & & 54.1 & \\
\hline
\end{tabular}

Table 2 (continued) 
Table 2 (continued)

\begin{tabular}{|c|c|c|c|c|c|}
\hline Variables & No. & \multicolumn{2}{|c|}{ Cancer-specific survival (CSS) } & \multicolumn{2}{|c|}{ Overall survival (OS) } \\
\hline Well/moderate & 5,821 & 85.1 & $<0.001$ & 67.9 & $<0.001$ \\
\hline Poor/undifferentiated & 3,118 & 81.8 & & 55.7 & \\
\hline Unknown & 641 & 86.3 & & 64.8 & \\
\hline$<3$ & 1,696 & 81.6 & 0.001 & 54.2 & $<0.001$ \\
\hline$\geq 3$ & 7,884 & 84.7 & & 65.8 & \\
\hline \multicolumn{6}{|l|}{ Radiation } \\
\hline No radiation & 9,419 & 78.9 & 0.408 & 64.2 & $<0.001$ \\
\hline Yes & 328 & 88.9 & 0.053 & 53.3 & $<0.001$ \\
\hline No/unknown & 9,252 & 84.0 & & 64.0 & \\
\hline \multicolumn{6}{|l|}{ Insurance } \\
\hline Insured & 5,406 & 85.7 & $<0.001$ & 69.5 & $<0.001$ \\
\hline Uninsured & 88 & 90.3 & & 65.8 & \\
\hline Unknown & 4,086 & 82.5 & & 58.0 & \\
\hline \multicolumn{6}{|l|}{ Marital status } \\
\hline Single & 3,770 & 82.3 & $<0.001$ & 61.0 & $<0.001$ \\
\hline
\end{tabular}

ADC, adenocarcinoma; SCC, squamous cell carcinoma; No., number.

sufficient margins and to examine more lymph nodes in this specific population $(1,15)$.

In 2011, Whitson et al. used the SEER database to compare survival after segmental resection versus lobectomy in patients diagnosed with pathological stage I adenocarcinoma (ADC) or squamous cell carcinoma (SCC) between 1998 and 2007 (10). After stratifying the patients by tumor size, they found that lobectomy was associated with more favorable CSS and OS for tumors measuring $21-30 \mathrm{~mm}$, which is corroborated by the results of our study (10). A more recent National Cancer Database (NCDB) study that reviewed 143,040 patients with cT1-3N0M0 NSCLC concluded that the survival advantage offered by lobectomy over segmental resection in patients with ADC $>10 \mathrm{~mm}$ and SCC $>15 \mathrm{~mm}$ (both categories encompass the size of interest of $21-30 \mathrm{~mm}$ in our study) could be confirmed in multivariate Cox regression models and subgroup analyses (16). However, in a larger single-institution retrospective cohort study of 145 cases, Carr et al. reported that there were no differences in mortality, recurrence, or CSS between the segmental resection group and the lobectomy group for confirmed T1bN0M0 (21-30 mm) NSCLC, staged according to the 7th edition of the TNM staging system (17). More recently, Chan et al. observed no differences in OS or RFS based on a comparison of segmental resection and 
A

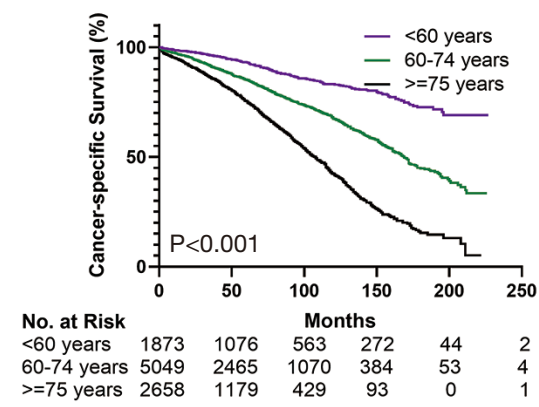

C

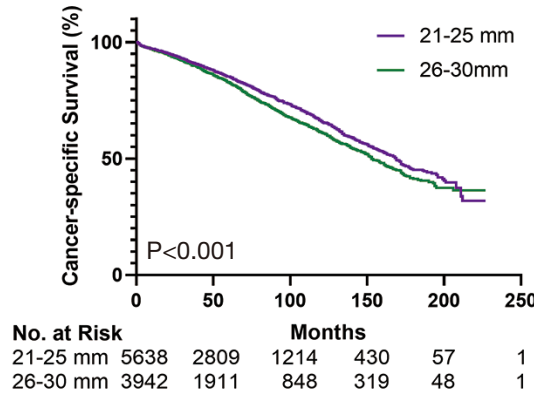

$E$

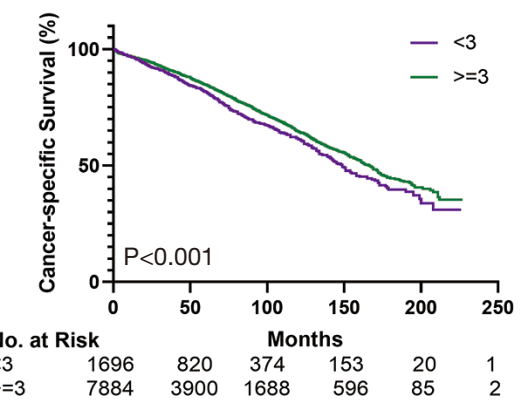

G

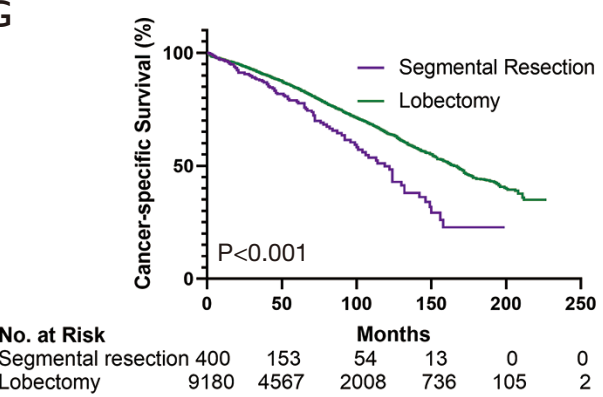

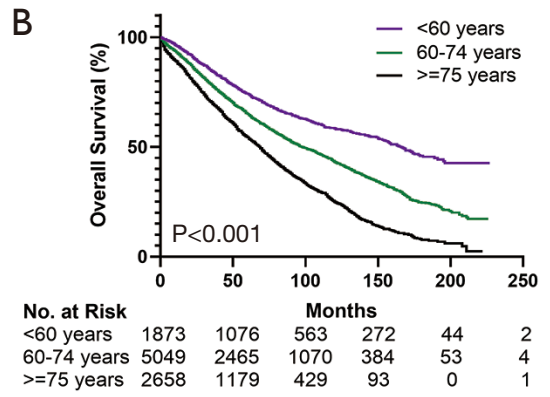

D

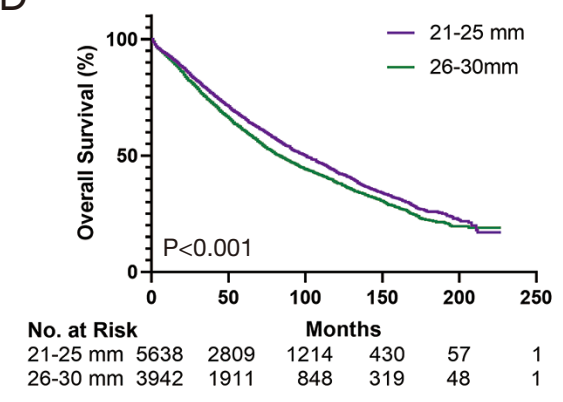

$\mathrm{F}$

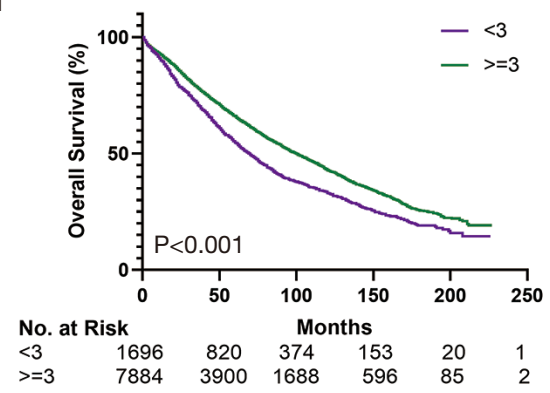

$\mathrm{H}$

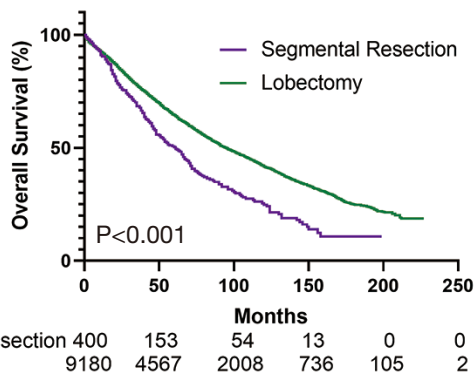

Figure 2 Survival analysis for patients with pathological stage IA $(21-30 \mathrm{~mm})$ NSCLC: CSS (A) and OS (B) comparison between groups according to age ( $<60 / 60-74 / \geq 75$ years); CSS (C) and OS (D) comparison between groups according to tumor size (21-25 $\mathrm{mm} / 26-30 \mathrm{~mm})$; CSS (E) and OS (F) comparison between groups according to the number of lymph nodes dissected $(<3 / \geq 3)$; and CSS $(\mathrm{G})$ and OS $(\mathrm{H})$ comparison between the segmental resection group and lobectomy group. CSS, cancer-specific survival; OS, overall survival; No., number.

lobectomy in 90 propensity score-matched pairs with clinical T1cN0M0 NSCLC (9). Subsequently, a Japanese singlecenter retrospective study also showed that the 33 matched pairs with radiologically solid-dominant clinical T1cN0M0 lung tumors who underwent lobectomy and segmental resection, had comparable OS and RFS (3). In spite of the evidence mentioned above, considerable controversy still surrounds the optimal extent of lung resection for clinical/ 
Table 3 (continued)

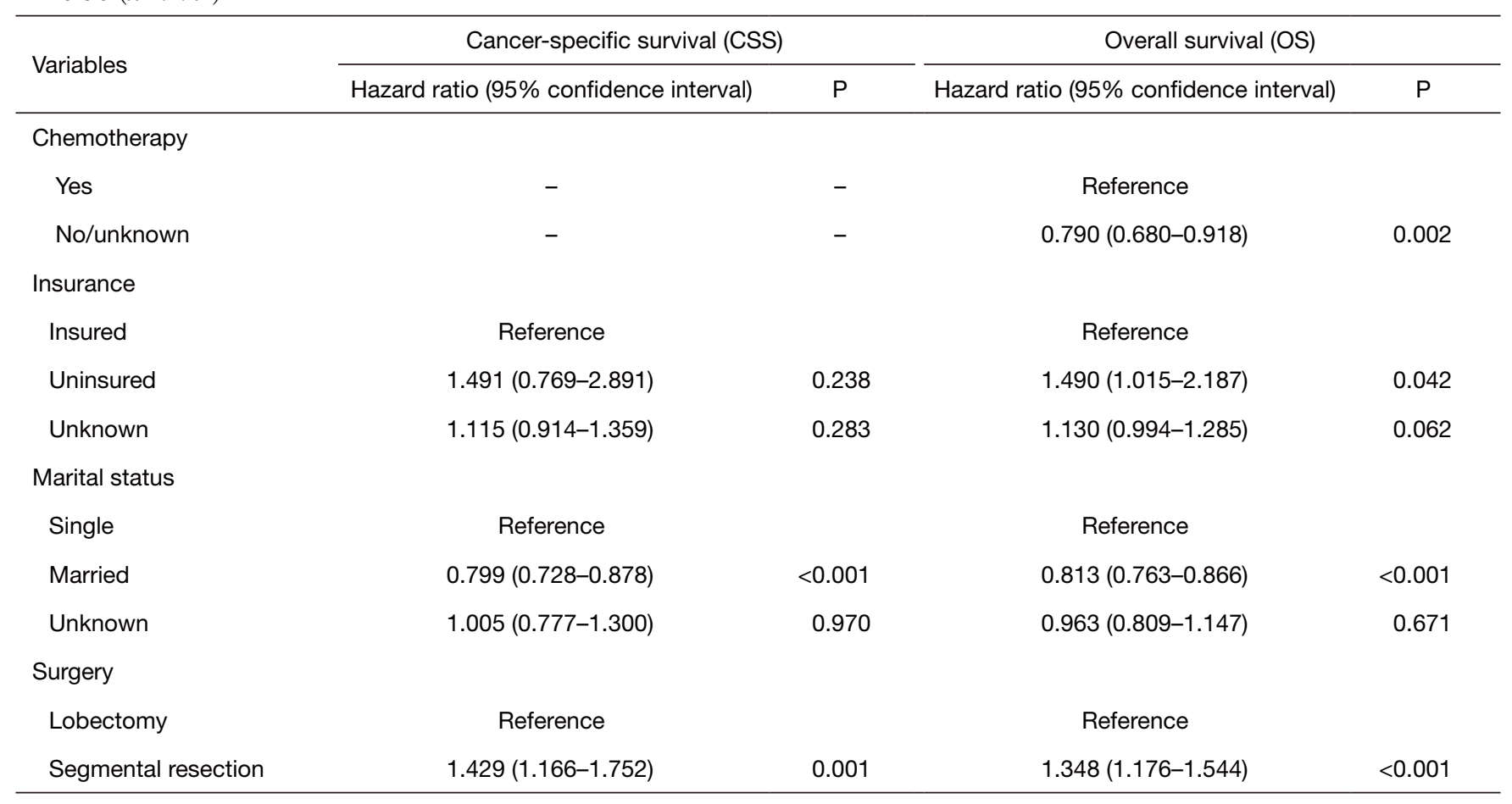

ADC, adenocarcinoma; SCC, squamous cell carcinoma; No., number.

pathological T1cN0M0 lung cancer. This may be due to the varied oncological outcomes from these small sample-sized, retrospective, single-center and population-based studies, or to the differences in the population choices and pathologies studied. Given this, prospective RCTs that consider the consolidation-to-tumor ratio should further assess the oncological performance of segmental resection for clinical stage IA lung tumors measuring 21-30 $\mathrm{mm}$. The present study thus represents a first, if not modest step, in this direction.

An additional advantage of lobectomy, compared to segmental resection, is that it allows the interlobar/hilar lymph nodes (N1) and mediastinal lymph nodes (N2) to be fully assessed to obtain more accurate pathological staging and possibly favorable survival $(9,11,17)$. In the present study, for patients with pathological stage IA (21-30 mm) NSCLC, a survival benefit was observed when the number of dissected lymph nodes was $\geq 3$, and the lobectomy group had consistent survival advantages compared to the segmental resection group when the patients were stratified by the number of dissected lymph nodes $(<3 v s$. $\geq 3)$. These findings support those of another SEER database study that compared segmental resection and lobectomy in patients with stage I $(\leq 30 \mathrm{~mm})$ NSCLC divided into three subgroups according to the analogous number of lymph nodes removed $(0 / 1-3 />3)$ (11). Moreover, many studies have reported that $>3$ lymph node stations or 10 dissected lymph nodes in clinical node-negative early-stage NSCLC not only significantly increases pathological nodal upstaging, but may also improve the long-term survival outcomes, regardless of whether segmental resection or lobectomy is used as treatment (18-20). Therefore, in consideration of the fact that a tumor size of $21-30 \mathrm{~mm}$ is a strong predictor of lymph node metastasis in cN0 NSCLC (14), a minimum of 3 lymph node stations or 10 lymph nodes should be dissected for this specific population during anatomical lung resection.

Additionally, we found that the proportion of patients who underwent radiation therapy after surgery in the segmental resection group was significantly higher than that in the lobectomy group $(3.5 \%$ vs. $1.6 \%, \mathrm{P}=0.004)$. Moreover, multivariate Cox regression analysis indicated that radiation after surgery for pathological stage IA $(21-30 \mathrm{~mm})$ NSCLC was associated with a worse OS, and the results from Qu et al.'s study support these findings (11). Although the SEER database contains no detailed information on the indication for postoperative radiotherapy in early-stage NSCLC, we hypothesized that radiation oncologist might 


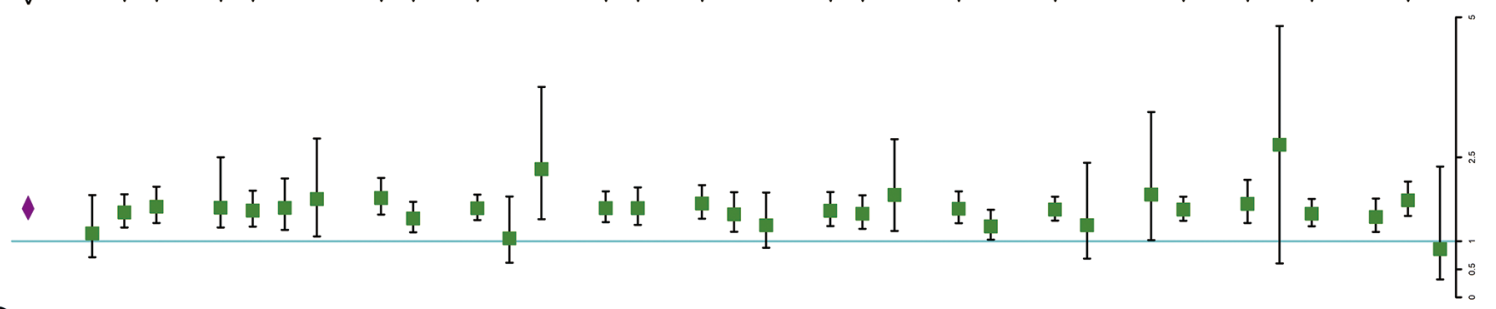

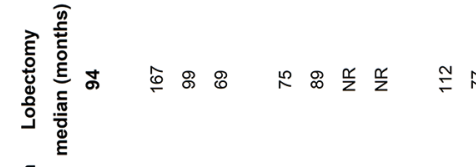

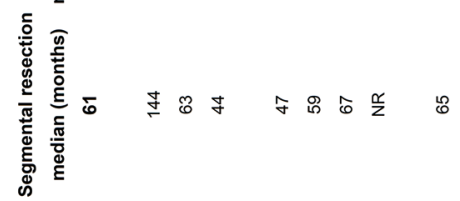


have recommended this multimodal therapy for cases with positive surgical margins, pathological high-risk factors, a lack of lymph node assessment, or local recurrence (6). Consequently, the present study provides further support that maintaining a safe surgical margin and implementing extended lymph node removal are important oncological principles in surgery for node-negative early-stage NSCLC.

This population-based study had several limitations, and its results should be interpreted with caution. First, this retrospective study cohort is abstracted from a national database and the years of diagnosis spans 19 years; this inevitably caused selection bias and attrition bias. Second, many studies have reported a high rate of local recurrence in patients who undergo segmental resection rather than lobectomy, which may result in poorer survival. However, information on disease recurrence status and RFS are not recorded in the SEER database. Third, there was a lack of information on comorbidities, physical status, QoL, and pulmonary function, which made it challenging to establish whether patients treated with segmental resection could also tolerate lobectomy; thus, selection bias might have affected the survival analyses. Fourth, several recent investigations have emphasized the value of the consolidation-to-tumor ratio $(\leq 0.25$ or $\leq 0.5)$ and pathological subtypes (micropapillary, solid, lepidic etc.) as important factors in choosing the surgical procedure. The lack of radiological and pathological data on the SEER database forced us to extract cases only on the basis of a tumor size measuring $21-30 \mathrm{~mm}$, and thus enrolling some part-solid nodules and high-risk subtypes was inevitable. The current results should be further verified through RCTs that factor in the consolidation-to-tumor ratio and pathological examination. Finally, the large range of subgroup sizes rendered any direct comparison between them difficult. Even the results in our study that reached statistical significance cannot preclude the possibility of group size imbalance which should also be considered when interpreting and drawing conclusions from these results.

In conclusion, as indicated by the relevant literature and guidelines, anatomical lobectomy with the extended removal of lymph nodes should be the standard of care for patients with resectable clinical/pathological T1cN0M0 NSCLC.

\section{Acknowledgments}

We thank Dr. A. Gillibert (Rouen University Hospital, Normandy, France) for reviewing the methodology and statistical analyses.

Funding: This study was funded by the National Key
Research and Development Plan (No. 2016YFC0905400) of the Ministry of Science and Technology of the People's Republic of China.

\section{Footnote}

Reporting Checklist: The authors have completed the STROBE reporting checklist. Available at http://dx.doi. org/10.21037/tlcr-20-1217

Conflicts of Interest: All authors have completed the ICMJE uniform disclosure form (available at http://dx.doi. org/10.21037/tlcr-20-1217). RHP reports that he received speaker fee from Medtronic. The other authors have no conflicts of interest to declare.

Ethical Statement: The authors are accountable for all aspects of the work in ensuring that questions related to the accuracy or integrity of any part of the work are appropriately investigated and resolved. The study was conducted in accordance with the Declaration of Helsinki (as revised in 2013). This retrospective study based on the SEER database was approved by the Surveillance Research Program in National Cancer Institute's Division of Cancer Control and Population Sciences (DCCPS) (No. 12101Nov2018), and was also approved by the institutional review at the Sun Yat-sen University Cancer Center (No. B2018011). Because of the retrospective nature of the research, the requirement for informed consent was waived.

Open Access Statement: This is an Open Access article distributed in accordance with the Creative Commons Attribution-NonCommercial-NoDerivs 4.0 International License (CC BY-NC-ND 4.0), which permits the noncommercial replication and distribution of the article with the strict proviso that no changes or edits are made and the original work is properly cited (including links to both the formal publication through the relevant DOI and the license). See: https://creativecommons.org/licenses/by-nc-nd/4.0/.

\section{References}

1. Goldstraw P, Chansky K, Crowley J, et al. The IASLC Lung Cancer Staging Project: Proposals for Revision of the TNM Stage Groupings in the Forthcoming (Eighth) Edition of the TNM Classification for Lung Cancer. J Thorac Oncol 2016;11:39-51.

2. Villamizar N, Swanson SJ. Lobectomy vs. segmentectomy 
for NSCLC ( $\mathrm{T}<2 \mathrm{~cm})$. Ann Cardiothorac Surg 2014;3:160-6.

3. Kamigaichi A, Tsutani Y, Kagimoto A, et al. Comparing Segmentectomy and Lobectomy for Clinical Stage IA Solid-dominant Lung Cancer Measuring 2.1 to $3 \mathrm{~cm}$. Clin Lung Cancer 2020;21:e528-e538.

4. Moon MH, Moon YK, Moon SW. Segmentectomy versus lobectomy in early non-small cell lung cancer of $2 \mathrm{~cm}$ or less in size: A population-based study. Respirology 2018;23:695-703.

5. Ginsberg RJ, Rubinstein LV. Randomized trial of lobectomy versus limited resection for T1 N0 non-small cell lung cancer. Lung Cancer Study Group. Ann Thorac Surg 1995;60:615-22; discussion 622-3.

6. Ettinger DS, Wood DE, Aggarwal C, et al. NCCN Guidelines Insights: Non-Small Cell Lung Cancer, Version 1.2020. J Natl Compr Canc Netw 2019;17:1464-72.

7. Nakamura K, Saji H, Nakajima R, et al. A phase III randomized trial of lobectomy versus limited resection for small-sized peripheral non-small cell lung cancer (JCOG0802/WJOG4607L). Jpn J Clin Oncol 2010;40:271-4.

8. Qiu B, Ji Y, He H, et al. Three-dimensional reconstruction/personalized three-dimensional printed model for thoracoscopic anatomical partial-lobectomy in stage I lung cancer: a retrospective study. Transl Lung Cancer Res 2020;9:1235-46.

9. Chan EG, Chan PG, Mazur SN, et al. Outcomes with segmentectomy versus lobectomy in patients with clinical T1cN0M0 non-small cell lung cancer. J Thorac Cardiovasc Surg 2020. doi: 10.1016/j.jtcvs.2020.03.041.

10. Whitson BA, Groth SS, Andrade RS, et al. Survival after lobectomy versus segmentectomy for stage I non-small cell lung cancer: a population-based analysis. Ann Thorac Surg 2011;92:1943-50.

11. Qu X, Wang K, Zhang T, et al. Long-term outcomes of stage I NSCLC $(\leq 3 \mathrm{~cm})$ patients following segmentectomy are equivalent to lobectomy under analogous extent of

Cite this article as: $\mathrm{Yu} \mathrm{X}$, Zhang $\mathrm{R}$, Zhang $\mathrm{M}$, Lin $\mathrm{Y}$, Zhang X, Wen Y, Yang L, Huang Z, Wang G, Zhao D, Gonzalez M, Baste JM, Petersen RH, Ng CSH, Brunelli A, Zheng L, Zhang L; written on behalf of the AME Thoracic Surgery Collaborative Group. Segmental resection is associated with decreased survival in patients with stage IA non-small cell lung cancer with a tumor size of $21-30 \mathrm{~mm}$. Transl Lung Cancer Res 2021;10(2):900-913. doi: 10.21037/tlcr-20-1217 lymph node removal: a PSM based analysis. J Thorac Dis 2017;9:4561-73.

12. Berfield KS, Wood DE. Sublobar resection for stage IA non-small cell lung cancer. J Thorac Dis 2017;9:S208-10.

13. Okada M, Nishio W, Sakamoto T, et al. Effect of tumor size on prognosis in patients with non-small cell lung cancer: the role of segmentectomy as a type of lesser resection. J Thorac Cardiovasc Surg 2005;129:87-93.

14. Tsutani Y, Miyata Y, Nakayama H, et al. Prediction of pathologic node-negative clinical stage IA lung adenocarcinoma for optimal candidates undergoing sublobar resection. J Thorac Cardiovasc Surg 2012;144:1365-71.

15. Nomori H, Mori T, Ikeda K, et al. Segmentectomy for selected cT1N0M0 non-small cell lung cancer: a prospective study at a single institute. J Thorac Cardiovasc Surg 2012;144:87-93.

16. Raman V, Jawitz OK, Voigt SL, et al. The Effect of Tumor Size and Histologic Findings on Outcomes After Segmentectomy vs Lobectomy for Clinically Node-Negative Non-Small Cell Lung Cancer. Chest 2021;159:390-400.

17. Carr SR, Schuchert MJ, Pennathur A, et al. Impact of tumor size on outcomes after anatomic lung resection for stage 1A non-small cell lung cancer based on the current staging system. J Thorac Cardiovasc Surg 2012;143:390-7.

18. Zhou H, Tapias LF, Gaissert HA, et al. Lymph Node Assessment and Impact on Survival in Video-Assisted Thoracoscopic Lobectomy or Segmentectomy. Ann Thorac Surg 2015;100:910-6.

19. Ludwig MS, Goodman M, Miller DL, et al. Postoperative survival and the number of lymph nodes sampled during resection of node-negative non-small cell lung cancer. Chest 2005;128:1545-50.

20. Zhao ZR, Situ DR, Lau RWH, et al. Comparison of Segmentectomy and Lobectomy in Stage IA Adenocarcinomas. J Thorac Oncol 2017;12:890-6. 